\title{
QUANTUM INSTABILITIES AND DECOHERENCE PROBLEM
}

\author{
S.Ya. Kilin, D.B. Horoshko and V.N. Shatokhin \\ Institute of Physics, Belarus Academy of Sciences \\ F. Skarina Avenue 70, Minsk 220072, Belarus
}

\begin{abstract}
A decoherence problem is discussed by means of quantum continuous measurement theory. It is shown that the conditional state of quantum system interacting with a bath preserves its initial purity. In this presentation decoherence arises as a result of averaging over the stochastic times of reduction moments ("clicks"). A method based on external phase feedback is proposed to slow down the decoherence of field superposition state in an open optical cavity. It is also shown that an atom placed inside the optical cavity plays a role of internal self-organized positive feedback between field and atom, which leads to an exponential increase in the mean dipole moment of the atom for the field initially prepared in a superposition of coherent states, i.e. to quantum instability.
\end{abstract}

PACS numbers: $42.50 . \mathrm{Lc}, 03.65 . \mathrm{Bz}$

\section{Introduction}

A decoherence problem is one of the fundamental problems of quantum theory. The problem has been formulated by E. Schrödinger in his famous "cat-state" paper [1] published in 1935. The problem was presented in a grotesque form which is perturbing our perception of the world. E. Schrödinger asked about possibility of observing macroscopic superposition of two states of a cat: alive $|\stackrel{0}{Q}\rangle$ and dead $|0 Q\rangle$. Why has such kind of macroscopic superposition never been registered in the real world? A reasonable answer for the question has been done by Zurek et al. in a series of papers [2-5]. Using the density matrix approach they argued about a rather common statement: the larger the cat size the faster the interference part of density matrix $(|\stackrel{0}{Q}\rangle\langle 0 Q|+| 0 Q\rangle\langle\stackrel{0}{Q}|)$ disappears, and the superposition evolves into a classical mixture. Decoherence being a very fundamental problem obtained recently a practical meaning because of limits put by it on the practical realization of quantum computers [6]. In this paper we discuss some new methods to influence fast decoherence and to slow it down. 


\section{External feedback and the decoherence problem}

There have been several proposals for slowing down the fast decoherence process in quantum systems [7-11]. Most of them are connected with Zurek's idea [2] that in the course of relaxation the quantum system is projected into a preferable basis ("pointer basis") formed via "quantum system - environment" interactions. Let us choose a harmonic oscillator as a quantum system interacting with the environment formed by a set of reservoir harmonic oscillators via an interaction Hamiltonian

$$
H_{\text {int }}=A\left(a, a^{+}\right) \Gamma^{+}+A^{+}\left(a, a^{+}\right) \Gamma,
$$

with $\Gamma=\hbar \sum_{i} g_{i} b_{i}$, where $A\left(a, a^{+}\right)$is a function of annihilation and creation operators of the selected oscillator, $g_{i}$ are coupling constants and $b_{i}, b_{i}^{+}$are annihilation and creation operators of the reservoir oscillators. In this case it becomes evident that for $A=A^{+}=R$ the eigenstates of $R$ form the pointer basis and they are not changed via interaction with environment. Therefore there is a possibility to select a different pointer basis by constructing a different form of coupling operator $A\left(a, a^{+}\right)$(the so-called "quantum reservoir engineering"). Some known examples are summarized in Table.

TABLE

Overview of different forms of system-reservoir coupling.

\begin{tabular}{l|l|l|l}
\hline \hline $\begin{array}{c}\text { Form of } \\
\text { coupling }\end{array}$ & \multicolumn{1}{|c|}{$\begin{array}{c}\text { Zurek's } \\
\text { "pointer basis" }\end{array}$} & $\begin{array}{c}\text { Steady } \\
\text { state }\end{array}$ & \multicolumn{1}{|c}{ References } \\
\hline$A=a+a^{+} \sim x$ & $\begin{array}{l}\text { position } \\
\text { eigenstates }\end{array}$ & vacuum & $\begin{array}{l}\text { Zurek et al. } \\
(1981,82,89,91) \\
\text { Caldeira, Leggett(1985) } \\
\text { Walls, Milburn (1985) }\end{array}$ \\
\hline$A=a^{2}$ & $\begin{array}{l}\text { even, odd } \\
\text { coherent states }\end{array}$ & vacuum & Gerry, Hach (1993) \\
\hline$A=(a+\alpha)(a-\alpha)$ & $\begin{array}{l}\text { even, odd } \\
\text { coherent states } \\
(\text { CS) }\end{array}$ & even, odd & $\begin{array}{l}\text { Garraway, Knight (1994) } \\
\text { Filho, W. Vogel (1996) } \\
\text { Poyatos, Zoller, Cirac } \\
\text { (1996) }\end{array}$ \\
\hline$A=a^{+} a$ & Fock states & vacuum & $\begin{array}{l}\text { Poyatos, Zoller, } \\
\text { Cirac (1996) }\end{array}$ \\
\hline$A=a\left(a^{+} a-n\right)$ & Fock states & Fock state & $\begin{array}{l}\text { Poyatos, Zoller, } \\
\text { Cirac (1996) }\end{array}$ \\
\hline \hline$A=\mathrm{e}^{\mathrm{i} \pi a^{+} a} a$ & Yurke-Stoler CS & vacuum & Horoshko, Kilin (1997) \\
\hline
\end{tabular}

In the present paper we discuss the decoherence problem for a specific initial state of the harmonic oscillator: a superposition of two coherent states with 
opposite phases

$$
\left|\psi_{+}\right\rangle=N\left(|\alpha\rangle+\mathrm{e}^{\mathrm{i} \theta}|-\alpha\rangle\right), \quad N^{-2}=2\left(1+\cos \theta \exp \left(-2|\alpha|^{2}\right)\right) .
$$

This state exhibits remarkable quantum features [12]. For instance, the Wigner function $W(\beta)$ of this state besides two positive peaks centered at $\beta= \pm \alpha$ has the interference part at $\beta=0$ which can take negative values. This nonpositive interference structure is a manifestation of quantum nature of the superposition (2). In the case of linear system-reservoir interaction $(A=a)$, the decoherence process consists in the fast disappearance of this interfeience part with the speed, which is $|\alpha|^{2}$ times higher than the speed of the peaks decay. The evolution of the density matrix averaged over the vacuum states of the reservoir in the Born-Markov approximation is described by the standard master equation

$$
\dot{\varrho}=\frac{\gamma}{2}\left(2 a \varrho a^{+}-a^{+} a \varrho-\varrho a^{+} a\right),
$$

where $\gamma=\pi \varrho(\omega)|g(\omega)|^{2}$ is the energy decay rate of the selected oscillator, $\varrho(\omega)$ is the density of reservoir states at the oscillator frequency $\omega$. The exact solution of Eq. (3) with the initial condition $\varrho(0)=\left|\psi_{+}\right\rangle\left\langle\psi_{+}\right|$has the following form:

$$
\begin{aligned}
\varrho(t) & =\frac{1}{2}\left(\left|\alpha_{t}\right\rangle\left\langle\alpha_{t}|+|-\alpha_{t}\right\rangle\left\langle-\alpha_{t}\right|\right) \\
& +\frac{1}{2} \mathrm{e}^{-2|\alpha|^{2}(1-\exp (-\gamma t))}\left(\mathrm{e}^{\mathrm{i} \theta}\left|-\alpha_{t}\right\rangle\left\langle\alpha_{t}\left|+\mathrm{e}^{-\mathrm{i} \theta}\right| \alpha_{t}\right\rangle\left\langle-\alpha_{t}\right|\right),
\end{aligned}
$$

where $\alpha_{t}=\alpha \mathrm{e}^{-\gamma t / 2}$. Expression (4) clearly shows the fast disappearance of the quantum interference terms with the rate of $t_{\text {decoh }}^{-1}=2 \gamma|\alpha|^{2}$. However, it does not allow us to understand all the details of the decoherence process. The most convenient and furthermore productive way for that is to use the continuous measurement theory [13]. Using this theory one can calculate the conditional state of the harmonic oscillator after emitting exactly $n$ quanta into the reservoir at stochastic times $t_{1}, t_{2}, \ldots, t_{n}$ in the time interval $[0, t)$

$$
\left|\psi_{\text {cond }}(t)\right\rangle=\gamma^{n} S\left(t, t_{n}\right) a S\left(t_{n}, t_{n-1}\right) a \ldots a S\left(t_{1}, 0\right)\left|\psi_{+}\right\rangle
$$

where $S\left(t_{i}, t_{i-1}\right)=\exp \left(-\gamma a^{+} a\left(t_{i}-t_{i-1}\right) / 2\right)$ is a nonunitary operator of evolution between two emission times $t_{i-1}$ and $t_{i}$ The emission of quanta at times $\left\{t_{i}\right\}$ makes a reduction of the system state according to action of the annihilation operator $a$. So, according to Eq. (5) and the relation

$$
a\left(|\alpha\rangle \pm \mathrm{e}^{\mathrm{i} \theta}|-\alpha\rangle\right)=\alpha\left(|\alpha\rangle \mp \mathrm{e}^{\mathrm{i} \theta}|-\alpha\rangle\right),
$$

the system changes the relative phase $\theta$ on $\pi$ at the moments of clicks but the state of the system remains a pure superposition state. Between the clicks the amplitude of the state diminishes according to non-unitary evolution operator action

$$
\begin{aligned}
& \exp \left(-\gamma a^{+} a\left(t_{i}-t_{i-1}\right) / 2\right)\left(|\alpha\rangle \pm \mathrm{e}^{\mathrm{i} \theta}|-\alpha\rangle\right) \\
& \quad=\left(\left|\alpha \mathrm{e}^{-\gamma\left(t_{i}-t_{i-1}\right) / 2}\right\rangle \pm \mathrm{e}^{\mathrm{i} \theta}\left|-\alpha \mathrm{e}^{-\gamma\left(t_{i}-t_{i-1}\right) / 2}\right\rangle\right) .
\end{aligned}
$$

Therefore, the stochastic history of the selected harmonic oscillator tells us that its state during all period of evolution is a superposition state with preserved coherence

$$
\left|\psi_{\text {cond }}(t)\right\rangle=N(\gamma \alpha)^{n}\left(\left|\alpha \mathrm{e}^{-\gamma t / 2}\right\rangle+(-1)^{n} \mathrm{e}^{\mathrm{i} \theta}\left|-\alpha \mathrm{e}^{-\gamma t / 2}\right\rangle\right)
$$


However if we want to discuss the unconditional state which suits for ensemble measurements, we should average the conditional density matrix $\left|\psi_{\text {cond }}(t)\right\rangle\left\langle\psi_{\text {cond }}(t)\right|$ over the stochastic times of clicks $\left\{t_{i}\right\}$. This averaging gives exactly the density matrix (4) which describes the loss of coherence which can be understood as the loss of information about the system state. From the continuous measurement theory representation it becomes evident that to erase the quantum interference terms it is enough to average over the first click time. And because the mean time of waiting for the first click is of the order of $1 /\left(\gamma|\alpha|^{2}\right)$, one can immediately obtain the decoherence time value $t_{\text {decoh }}=1 /\left(\gamma|\alpha|^{2}\right)$. Another lesson which follows from the consideration is that there is a way for slowing down the decoherence: after each click it is necessary to change back the relative phase in the superposition of coherent states.

We can also come to the same conclusion using the quantum engineering approach [11]. As is shown in this paper, the generalized annihilation and creation operators

$$
A_{\varphi}=\mathrm{e}^{\mathrm{i} \varphi a^{+} a} a, \quad A_{\varphi}^{+}=a^{+} \mathrm{e}^{-\mathrm{i} \varphi a^{+} a}
$$

obey boson commutation relations

$$
\left[A_{\varphi}, A_{\varphi}^{+}\right]=1
$$

and, what is more useful, the eigenstates of the operator $A_{\varphi}$

$$
A_{\varphi}\left|\mathcal{A}_{\varphi}\right\rangle=\mathcal{A}_{\varphi}\left|\mathcal{A}_{\varphi}\right\rangle
$$

are the states with Poissonian distribution of photons, but with quadratic dependence of amplitude phase

$$
\left|\mathcal{A}_{\varphi}\right\rangle=\mathrm{e}^{-\left|\mathcal{A}_{\varphi}\right|^{2} / 2} \sum_{n} \frac{\mathcal{A}_{\varphi}^{n}}{\sqrt{n !}} \mathrm{e}^{\mathrm{i} \varphi n(n-1) / 2}|n\rangle .
$$

These states belong to the class of states, firstly introduced by Glauber and Titulaer in Ref. [14], where they discussed the states with all degrees of coherence equal to unity. Note that the class of states (9) has a number of unusual quantum properties. As an example we demonstrate the $Q$-function of $\left|\mathcal{A}_{2 \pi / 7}\right\rangle$-state in Fig. 1. The $\left|\mathcal{A}_{\pi}\right\rangle$-state coincides with the Yurke-Stoler state given by Eq. (2) with $\theta=\pi / 2, \mathcal{A}_{\pi}=\mathrm{i} \alpha$. Taking into account this fact, we use $A_{\pi}$ as a nonlinear coupling operator in interaction Hamiltonian (1) and obtain the following master equation:

$$
\dot{\varrho}=\frac{\gamma}{2}\left(\left[A_{\pi}, \varrho A_{\pi}^{+}\right]+\left[A_{\pi} \varrho, A_{\pi}^{+}\right]\right) .
$$

Being rewritten in a different form

$$
\dot{\varrho}=\frac{\gamma}{2}\left(2 \mathrm{e}^{\mathrm{i} \pi a^{+} a} a \varrho a^{+} \mathrm{e}^{-\mathrm{i} \pi a^{+} a}-a^{+} a \varrho-\varrho a^{+} a\right)
$$

this master equation is exactly the one for system under measurement mediated feedback. The evolution introduced by the first right-hand term is the state reduction (due to emission of quantum) followed by feedback action corresponding to interactive field phase changing on $\pi$. And now the conditional state evolution is described by the following equation:

$$
\left|\psi_{\text {cond }}(t)\right\rangle=\gamma^{n} S\left(t, t_{n}\right) \mathrm{e}^{\mathrm{i} \pi a^{+} a} a S\left(t_{n}, t_{n-1}\right) \mathrm{e}^{\mathrm{i} \pi a^{+} a} a \ldots \mathrm{e}^{\mathrm{i} \pi a^{+} a} a S\left(t_{1}, 0\right)\left|\psi_{+}\right\rangle,
$$




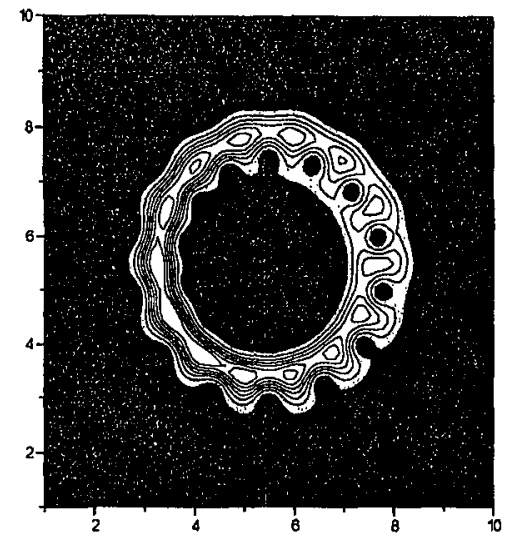

(a)

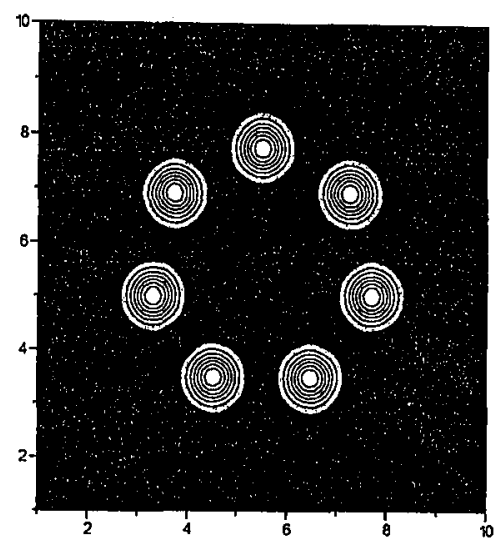

(b)

Fig. 1. $Q$-function (view from above) of the generalized coherent state given by Eq. (12) for $\alpha=5$ and (a) $\varphi=\pi / 7$, (b) $\varphi=2 \pi / 7$.

rather than Eq. (5). From this equation we easily find that this conditional state

$$
\left|\psi_{\text {cond }}(t)\right\rangle=\frac{1}{\sqrt{2}}(\gamma \alpha)^{n}\left(\left|\alpha \mathrm{e}^{-\gamma t / 2}\right\rangle+\mathrm{i}\left|-\alpha \mathrm{e}^{-\gamma t / 2}\right\rangle\right) \text {, }
$$

remains a superposition with a fixed relative phase. So, averaging over the stochastic times $\left\{t_{i}\right\}$ does not influence this statement, and the unconditional state in this case will be a pure superposition

$$
|\psi(t)\rangle=\frac{1}{\sqrt{2}}\left(\left|\alpha \mathrm{e}^{-\gamma t / 2}\right\rangle+\mathrm{i}\left|-\alpha \mathrm{e}^{-\gamma t / 2}\right\rangle\right) .
$$

Therefore a simple experimental scheme for slowing down the decoherence or for improving error introduced by decay processes could be proposed [11] as follows: after each successful click of the photon detector collecting all the light escaping the optical cavity one should use this information and change the intracavity field phase by $\pi$. Repeating this procedure we will allow the cat state $\left|\psi_{+}\right\rangle$for $\theta=\pi / 2$ to be a superposition as long as there are photons in the cavity*.

\section{Quantum instabilities, entanglement and feedback action of an atom in a high $Q$ cavity}

As is seen from the previous consideration, the intracavity field initially prepared in a superposition (2) will evolve to a mixture in a fast mode. So, in an empty cavity after a time larger than $t_{\text {decoh }}$ the state of the field will be described by density matrix

$$
\varrho_{\text {mix }}=\frac{1}{2}(|\alpha\rangle\langle\alpha|+|-\alpha\rangle\langle-\alpha|)
$$

without any quantum features. From the first sight the time $t_{\mathrm{decoh}}=1 /\left(\gamma|\alpha|^{2}\right)$ will limit all possible manifestations of quantum peculiarities of superposition state (2).

\footnotetext{
${ }^{*}$ A technical aspect of the proposed scheme should be noted: the feedback loop action time $\tau_{\mathrm{FB}}$ should be less than the a verage time between two successive clicks, which is of order $t_{\text {decoh }}=$ $1 /\left(\gamma|\alpha|^{2}\right)$. Therefore this fast switching of the intracavity field phase can put some problems with the single mode operation of the cavity.
} 
Below we show that a two-level atom placed inside cavity acts as internal positive feedback and this action leads to a quantum instability $[15,16]$ as well as to more prolonged period for field coherence manifestation.

As was shown in Ref. [15] the Glauber $P$-function of state (2) has four terms

$$
\begin{aligned}
P(\beta) & =N_{+}\left\{\delta^{(2)}(\alpha-\beta)+\delta^{(2)}(\alpha+\beta)+\left[\mathrm{e}^{-2|\alpha|^{2}} /|\beta|\right] \delta\left(\varphi_{\alpha}-\varphi_{\beta}+\pi / 2\right)\right. \\
& \left.\times\left[\mathrm{e}^{\mathrm{i} \theta} \delta_{\mathrm{AC}}(|\beta|-\mathrm{i}|\alpha|)+\mathrm{e}^{-\mathrm{i} \theta} \delta_{\mathrm{AC}}(|\beta|+\mathrm{i}|\alpha|)\right]\right\} .
\end{aligned}
$$

The first two terms correspond to a classical part. The latter terms describing the quantum interference part of this state are proportional to the so-called delta-functions of analytic continuation $\delta_{\mathrm{AC}}(|\beta| \pm i|\alpha|)$. The $\delta$-function of analytic continuation may be defined by its action on a probe function $F(x)$

$$
\int_{-\infty}^{\infty} \mathrm{d} x \delta_{\mathrm{AC}}(x-z) F(x)=F(z), x \in R^{1}, z \in C^{1} .
$$

The well-known solution for a mean dipole moment of a two-level atom in a free space immediately leads to an exponentially divergent solution when averaged with this function. We call this effect quantum instability [15] because of the reason of the effect: the quantum interference part of the density matrix of state (2).

The effect of the quantum instability may be observable in a high- $Q$ cavity at the initial part of evolution. If we use the Jaynes-Cummings $(\mathrm{J}-\mathrm{C})$ model the mean dipole moment of a two-level atom is (for an atom initially prepared in the ground state and the field in a state $|\psi\rangle)$

$$
\left\langle\sigma_{-}(t)\right\rangle=-\mathrm{i}\left\langle\psi\left|\cos (g t \sqrt{\widehat{n}}) \frac{\sin (g t \sqrt{\hat{n}+1})}{\sqrt{\hat{n}+1}} a\right| \psi\right\rangle,
$$

where $\widehat{n}=a^{+} a, g$ is the atom-field coupling constant. For initial coherent field state $|\psi\rangle=|\alpha\rangle$ Eq. (21) gives

$$
\left\langle\sigma_{-}(t)\right\rangle_{\mathrm{coh}}=-\mathrm{i} \alpha \mathrm{e}^{-|\alpha|^{2}} \sum_{k} \frac{|\alpha|^{2 k}}{k ! \sqrt{k+1}} \cos (g t \sqrt{k}) \sin (g t \sqrt{k+1}),
$$

while for the Yurke-Stoler superposition state $(\theta=\pi / 2)$

$$
\left\langle\sigma_{-}(t)\right\rangle_{\mathrm{YS}}=\alpha \mathrm{e}^{-2|\alpha|^{2}} \sum_{k} \frac{\left(-|\alpha|^{2}\right)^{k}}{k ! \sqrt{k+1}} \cos (g t \sqrt{k}) \sin (g t \sqrt{k+1}) .
$$

For the mixture (18) of coherent states $\left\langle\sigma_{-}(t)\right\rangle_{\text {mix }}=0$ for all moments of time. The main difference between Eq. (22) and Eq. (23) is that the Fourier components of the sum (23) have alternating sign in contrast to that of the sum (22). For large values of coherent amplitude $|\alpha|$ when the spacing $g(\sqrt{k+1}-\sqrt{k}) \approx g /(2 \sqrt{k}) \approx$ $g /(2|\alpha|)$ between significant components of the sum much less than the width $2|\alpha| g$ of the components distribution, the asymptotic of Eq. (23) is

$$
\left\langle\sigma_{-}(t)\right\rangle \approx \frac{1}{4 \sqrt{1-g t /(2|\alpha|)}} \mathrm{e}^{-2|\alpha|^{2}+2|\alpha| g t-(g t / 2)^{2}}
$$

instead of Rabi oscillation for Eq. (22). So, the exponential growth of the mean dipole moment for times $t \ll 2 \alpha / g$, which are smaller than revivals time $T_{\mathrm{R}}=$ $2 \pi \alpha / g$ is also observable for the $\mathrm{J}-\mathrm{C}$ model. 
A physical reason for this instability is entanglement between atomic and field states [15, 17]. As it has been shown in Ref. [15] the phase of interference part of the conditional Wigner function of the field follows the atomic state: the phase changes by $\pi$ when atom makes a transition from the ground state $|g\rangle$ to excited $|e\rangle$ and vice versa. It can be easily seen from the time-evolved atom-field state for initial state $|\psi(0)\rangle=|g\rangle\left|\psi_{+}\right\rangle$

$$
|\psi(t)\rangle=\cos (g t \sqrt{n})\left|\psi_{+}\right\rangle|g\rangle-\mathrm{i} \frac{\sin (g t \sqrt{n+1})}{\sqrt{n+1}}\left|\psi_{-}\right\rangle|e\rangle .
$$

So we can conclude that the quantum positive feedback (entanglement) between the phase of interference part of field Wigner function and state of two-level atom is the factor which leads to the quantum instability of the mean dipole moment.

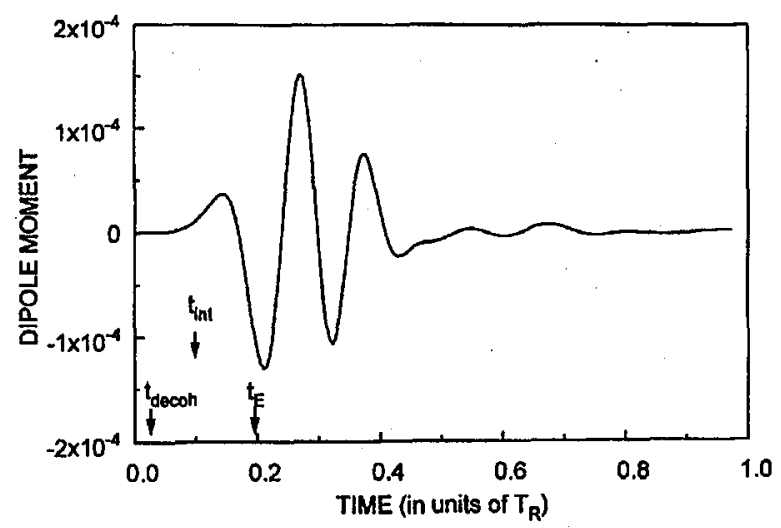

Fig. 2. Temporal dependence of the mean dipole moment in the optical resonator with the parameters $g=2 \pi \times 2.7 \mathrm{MHz}, k=2 \pi \times 0.7 \mathrm{MHz}, \gamma=2 \pi \times 2.5 \mathrm{MHz}$ for the initial superposition state $|\alpha\rangle+\mathrm{i}|-\alpha\rangle$ with $\alpha=3$. Arrows mark the decoherence time $t_{\mathrm{decoh}}=1 /\left(k \alpha^{2}\right)$, the interaction time $t_{\text {int }}$ and the time of the field energy decay $t_{\mathrm{E}}=1 / k$.

When a cavity damping is included into consideration, the fast decoherence processes should diminish all manifestations of coherent interactions. However atom seating in cavity sees continuously the state of the field, and as we demonstrated in Sec. 2, the continuous state of the field is nothing more than a pure superposition state with a changing relative phase at the moments when a photon escapes the cavity. That is why one could observe the effect of quantum instability at the initial stage of a two-level atom interaction with damping cavity mode prepared in the superposition state (2). The solution of master equation for the Jaynes-Cummings model with cavity decay $[16,18]$ supports this statement (see Fig. 2).

\section{Conclusions}

On the basis of quantum continuous measurement theory we demonstrated that the decoherence arises as a result of combination of averaging over stochastic 
times of the clicks due to the quanta passing into a bath, and reduction of the system state which occurs at these times. Without averaging the single-run-state of the decaying system is a pure quantum state. We showed that because of this purity the decoherence processes can be slowed down by adding corrections after reduction which each click makes. We proposed the concrete scheme for slowing down the decoherence of the Yurke-Stoler state on the basis of phase feedback which introduces a $\pi$ phase shift after detecting each photon escaping the cavity.

We also showed that a two-level atom placed inside a cavity plays a role of an internal positive feedback (in other words, entanglement) which leads to the effect of the quantum instability of the mean dipole moment of an atom. The exponential growth of the dipole moment can be observed at the initial stage of evolution of an atom in a cavity containing field prepared in a superposition state.

The authors gratefully acknowledge financial support by INTAS under grant RFBR 95-0656 and Belarus Foundation for Basic Researches under grants F205 and MP 96-38.

\section{References}

[1] E. Schrödinger, Naturwissenschaften 23, 807 (1935).

[2] W.H. Zurek, Phys. Today 44, 36 (1991).

[3] W.H. Zurek, Phys. Rev. D 24, 1516 (1981).

[4] W.H. Zurek, Phys. Rev. D 26, 1862 (1982).

[5] W.G. Unruh, W.H. Zurek, Phys. Rev. D 40, 1071 (1989).

[6] D.P. DiVincenzo, Science 270, 255 (1995).

[7] C. Gerry, E.E. Hach, Phys. Lett. A 174, 185 (1993).

[8] B.R. Garraway, P.L. Knight, Phys. Rev. A 49, 1266 (1994); ibid. 50, 2548 (1994).

[9] M.R.L. Filho, W. Vogel, Phys. Rev. Lett. 76, 608 (1996).

[10] J.F. Poyatos, J.I. Cirac, P. Zoller, Phys. Rev. Lett. 77, 4728 (1996).

[11] D.B. Horoshko, S.Ya. Kilin, Phys. Rev. Lett. 78, 840 (1997).

[12] W. Schleich, M. Pernigo, Fam Le Kien, Phys. Rev. A 44, 2172 (1991).

[13] E.B. Davies, Quantum Theory of Open Systems, Academic Press, New York 1976; S.Ya. Kilin, Quantum Optics: Fields and Detection of them, Nauka i Tekhnika, Minsk 1990 (in Russian).

[14] V.M. Titulaer, R.J. Glauber, Phys. Rev. A 145, 1046 (1986).

[15] S.Ya. Kilin, V.N. Shatokhin, Phys. Rev. Lett. 76, 1051 (1996).

[16] S.Ya. Kilin, V.N. Shatokhin, Opt. Spectrosc. 82, 972 (1997).

[17] S.J.D. Phoenix, P.L. Knight, Phys. Rev. A 44, 6023 (1991).

[18] J.H. Kimble, in: Advances in Atomic, Molecular and Optical Physics, Ed. P. Berman, Suppl. 2. Academic Press, New York 1994, p. 203. 